\title{
Camellia Plant Resistance and Susceptibility to Petal Blight Disease Are Defined by the Timing of Defense Responses
}

\author{
Nikolai Kondratev, ${ }^{1,2}$ Matthew Denton-Giles, ${ }^{1}$ Rosie E. Bradshaw, ${ }^{1,2}$ Murray P. Cox, ${ }^{1,2}$ and \\ Paul P. Dijkwel, ${ }^{1,2, \dagger}$ \\ ${ }^{1}$ School of Fundamental Sciences, Massey University, Palmerston North, New Zealand \\ ${ }^{2}$ Bio-Protection Research Centre, Massey University, Palmerston North, New Zealand
}

Accepted 23 March 2020.

The family Sclerotiniaceae includes important phytopathogens, such as Botrytis cinerea and Sclerotinia sclerotiorum, that activate plant immune responses to facilitate infection propagation. The mechanisms of plant resistance to these necrotrophic pathogens are still poorly understood. To discover mechanisms of resistance, we used the Ciborinia camelliae (Sclerotiniaceae)-Camellia spp. pathosystem. This fungus induces rapid infection of the blooms of susceptible cultivar Nicky Crisp (Camellia japonica $\times$ Camellia pitardii var. pitardii), while Camellia lutchuensis is highly resistant. Genome-wide analysis of gene expression in resistant plants revealed fast modulation of host transcriptional activity $6 \mathrm{~h}$ after ascospore inoculation. Ascospores induced the same defense pathways in the susceptible Camellia cultivar but much delayed and coinciding with disease development. We next tested the hypothesis that differences in defense timing influences disease outcome. We induced early defense in the susceptible cultivar using methyl jasmonate and this strongly reduced disease development. Conversely, delaying the response in the resistant species, by infecting it with actively growing fungal mycelium, increased susceptibility. The same plant defense pathways, therefore, contribute to both resistance and susceptibility, suggesting that defense timing is a critical factor in plant health, and resistance against necrotrophic pathogens may occur during the initial biotrophy-like stages.

Keywords: Camellia, defense signaling pathways, necrotrophy, plant antifungal responses, plant responses to pathogens, resistance, susceptibility, transcriptomics

The original read datasets that were used for Camellia cultivar Nicky Crisp reference transcriptome assembly, Camellia lutchuensis reference transcriptome assembly, and C. lutchuensis differential gene expression analysis are available in the National Center for Biotechnology Information database with BioProject accession numbers PRJNA518146, PRJNA518136, PRJNA506291, respectively.

${ }^{\dagger}$ Corresponding author: P. P. Dijkwel; P.Dijkwel@massey.ac.nz

Funding: This work was supported by the New Zealand Camellia Memorial Trust.

*The $\boldsymbol{e}$-Xtra logo stands for "electronic extra" and indicates that two supplementary figures and six supplementary tables are published online.

The author(s) declare no conflict of interest.

(c) 2020 The American Phytopathological Society
Plants from the genus Camellia have been cultivated and traded since ancient times ( $\mathrm{Lu}$ et al. 2016). In addition to being highly prized ornamental plants, comprising about 300 species and more than 3,000 cultivated varieties (Mondal 2011), camellias are used to produce the nonalcoholic beverage tea (Camellia sinensis) as well as seasoning and cooking oils (mainly Camellia oleifera and Camellia japonica). Many Camellia species and cultivars are susceptible to the necrotrophic fungal pathogen Ciborinia camelliae from the family Sclerotiniaceae, which causes petal blight disease. The main effect of this disease is the premature fall of blooms, which can reduce plant reproduction via a decrease in fruit production (Hara 1919). The disease originated in East Asia and quickly spread throughout the world due to plant trade and wind-borne ascospores (Taylor and Long 2000). Unfortunately, neither the application of chemical fungicides nor the use of various biocontrol agents have been successful in limiting infection rates or disease symptoms to levels that are tolerable by camellia growers (Saracchi et al. 2018).

Ciborinia camelliae is closely related to two devastating necrotrophic pathogens from the same family Sclerotiniaceae, namely, Botrytis cinerea and Sclerotinia sclerotiorum, which cause gray and white mold diseases, respectively, in multiple plant species (Kohn and Nagasawa 1984). Despite its close phylogenetic relationship to fungi whose infections are not specific to particular plant organs, Ciborinia camelliae infects only the floral organs of some Camellia species and hybrids, rapidly occupying the whole flower within just days (DentonGiles et al. 2013).

In earlier research on cellular defense mechanisms, Camellia species and cultivars were shown to exhibit various levels of susceptibility and resistance to petal blight, enabling preferred candidate species and cultivars to be selected for disease resistance breeding. Microscopic analysis showed that the incompatible interaction observed for Camellia lutchuensis and C. transnokoensis-the most resistant Camellia species tested-includes physiological processes like localized cell death, apposition of papillae, and hydrogen peroxide production (Denton-Giles et al. 2013). In contrast, the most susceptible Camellia, i.e., cultivar Nicky Crisp (Camellia japonica $\times$ Camellia pitardii var. pitardii), showed no resistance features. In tea crops, both $C$. sinensis var. sinensis and $C$. sinensis var. assamica have been shown to form a compatible interaction with Ciborinia camelliae (Denton-Giles et al. 2013). 
Fungal pathogens can be classified into three groups: biotrophs that acquire nutrients from living plant tissues, necrotrophs that grow on dead tissue, and hemibiotrophs that shift their growth strategy from biotrophic to necrotrophic during development. This variation in infection strategies forces plants to exploit multiple defense mechanisms. Plant resistance can be considered as a multistep process that includes i) recognition of the pathogen by pattern-recognition receptors or resistance $(\mathrm{R})$ proteins (immune receptors), ii) activation of multiple cellular signaling networks, and iii) activation of biochemical and cellular defense processes (Katagiri and Tsuda 2010). Transcriptomic studies of teapathogen interactions show that $\mathrm{R}$ protein immune receptors and transcription factors are master regulators of tea plant defense against the blister blight pathogen Exobasidium vexans (Jayaswall et al. 2016) and that reactive oxygen species facilitate the resistance to anthracnose, caused by Colletotrichum fruticola (Wang et al. 2018a).

While activation of defense pathways prevents the development of biotrophic pathogens, perversely, cell deathmediated plant immunity can be deliberately stimulated by necrotrophs to expand the necrotic area and hence enhance colonization (Wang et al. 2014). At the same time, resistance to necrotrophic pathogens still requires sophisticated and coordinated activation of multiple defense mechanisms, such as jasmonate- and ethylene-signaling (Glazebrook 2005), mitogen-activated protein kinase cascades (Li et al. 2014), and the production of secondary metabolites like glycosinolates (Wu et al. 2016), phenylpropanoids (Xu et al. 2011), terpenoids (De Cremer et al. 2013), and camalexin (Ferrari et al. 2003). The complexity of plant-fungal interactions makes it challenging to select target defense mechanisms for molecular resistance breeding.

Here, we report the plant-fungus interaction between $\mathrm{Ca}$ mellia spp. and Ciborinia camelliae. To identify transcriptional mechanisms underpinning the resistance of Camellia plants to Ciborinia camelliae, we sequenced and assembled transcriptomes of petal blight-resistant $C$. lutchuensis, and petal blight-susceptible $C$. japonica $\times C$. pitardii var. pitardii Nicky Crisp. We analyzed gene expression patterns associated with the resistance response to Ciborinia camelliae and found candidate regulator genes of the defense process as well as genes belonging to secondary metabolic pathways that may also contribute to resistance. Further analysis of the susceptible interaction led us to conclude that the timing of the defense response is a key factor defining the outcome of necrotrophic petal blight disease.

\section{RESULTS}

Sequencing and assembly of reference Camellia transcriptomes.

De novo assembly of short-read RNA sequences is widely used to build transcriptomes for non-model organisms that lack reference genomes. We generated transcriptomes for two $\mathrm{Ca}$ mellia plants that differ in their response to the petal blight pathogen Ciborinia camelliae: petal blight-resistant $C$. lutchuensis and petal blight-susceptible Camellia japonica $\times$ Camellia pitardii var. pitardii Nicky Crisp. RNA from mockinfected and infected petals was extracted and sequenced, generating over 100 million high-quality Illumina $2 \times 100$-bp paired-end reads for each of the resistant and susceptible plants (National Center for Biotechnology Information [NCBI] BioProjects PRJNA518136 and PRJNA518146). Since reads from infected petals had mixed species origins, sequences that mapped onto the Ciborinia camelliae genome (GenBank GCA_001247705.1) were removed. The remaining reads were assembled using the Trinity de novo assembler (Haas et al. 2013), resulting in C. lutchuensis and Camellia 'Nicky Crisp' floral transcriptomes of 109,201 and 133,997 assembled transcripts, respectively. A flowchart of the assembly process is presented in Supplementary Fig. S1.

To confirm the quality of the assembled transcriptomes, we first measured $\mathrm{N}_{50}$ values (1,204 for C. lutchuensis and 1,076 for Camellia 'Nicky Crisp') and average GC content (40.4\% for C. lutchuensis, 39.9\% for Camellia 'Nicky Crisp'). These values are similar to published Camellia transcriptomes (Fan et al. 2015; Xia et al. 2014; Yao et al. 2016). The gene-content completeness of the transcriptomes was assessed using BUSCO, which characterizes the presence of 'universal' singlecopy plant gene orthologs (Simão et al. 2015). A total of 86 and $89 \%$ of these genes were present in the C. lutchuensis and Camellia 'Nicky Crisp' transcriptomes (Table 1), respectively, suggesting that most genes present in both species were successfully sequenced and assembled.

\section{Transcriptome annotations and prediction of Camellia proteomes.}

Functional annotations were predicted based on matches to NCBI and Swiss-Prot databases (Table 1). Gene Ontology (GO) terms were assigned to 30,935 transcripts of resistant C. lutchuensis and 23,783 transcripts of susceptible Camellia 'Nicky Crisp'. Protein coding sequences were predicted with TransDecoder (Haas et al. 2013), resulting in 37,239 predicted protein sequences for C. lutchuensis and 40,404 for Camellia

Table 1. Assembly and annotation results

\begin{tabular}{|c|c|c|}
\hline Assembly and annotation statistics & Camellia lutchuensis & Camellia 'Nicky Crisp' \\
\hline Number of high-quality read-pairs used (millions) & 110 & 162 \\
\hline \multicolumn{3}{|l|}{ Assembly statistics } \\
\hline Number of assembled transcripts & 109,291 & 133,997 \\
\hline Transcript length range $(\mathrm{bp})$ & 201 to 14,732 & 201 to 15,756 \\
\hline $\mathrm{N}_{50}$ & 1,204 & 1,076 \\
\hline$\% \mathrm{GC}$ & 40.4 & 39.9 \\
\hline \multicolumn{3}{|l|}{ BUSCO completeness } \\
\hline Complete $(\%)$ & 80.0 & 82.3 \\
\hline Duplicated (\%) & 15.1 & 15.6 \\
\hline Fragmented $(\%)$ & 6.0 & 6.4 \\
\hline Missing $(\%)$ & 14.0 & 11.3 \\
\hline \multicolumn{3}{|l|}{ Annotation results } \\
\hline With nt hits & 35,876 & 39,927 \\
\hline With Swiss-Prot hits & 33,939 & 36,737 \\
\hline With GO terms & 30,935 & 23,783 \\
\hline Number of predicted proteins & 37,239 & 40,404 \\
\hline
\end{tabular}


'Nicky Crisp'. Orthologous proteins were predicted by comparison with the reference proteome of the well-studied model plant Arabidopsis thaliana (UniProt UP000006548). The two Camellia transcriptomes shared 10,125 orthology groups with A. thaliana, and an additional 8,410 orthology groups were shared between the two Camellia transcriptomes alone. Over half of $A$. thaliana proteins (56\%) could not be assigned to any predicted orthology group, while 38 and $41 \%$ remained unclassified in the lesser studied $C$. lutchuensis and Camellia 'Nicky Crisp' (Fig. 1).

\section{Inoculation of Ciborinia camelliae ascospores induces} rapid transcriptional responses in resistant $C$. lutchuensis.

To determine the transcriptional resistance response of C. lutchuensis following Ciborinia camelliae infection, we performed a quantitative RNA-seq experiment. RNA was extracted from $C$. lutchuensis petals collected at $0,6,12$, and $24 \mathrm{~h}$ after spraying with fungal spores and at 12 and $24 \mathrm{~h}$ after spraying with sterile distilled water (mock infection) (Fig. 2A). Approximately 9 to 12 million high-quality $2 \times 125$-bp pairedend reads from each sample (NCBI BioProject PRNA506291) were mapped to the fungal and resistant plant reference transcriptomes described above, with mapping rates reaching 95 to $97 \%$ (Supplementary Table S1). The proportion of fungal reads increased from 0 to $6 \mathrm{~h}$ postinoculation (hpi), before decreasing again to preinfection levels at $24 \mathrm{hpi}$ (Fig. 2B). Principal component analysis (PCA) of transcript read counts showed that the plant underwent dramatic transcriptional reprogramming (Fig. 2C). Biological triplicates cluster on the PCA graph, emphasizing the consistency of the response to fungal treatment. The biggest transcriptional changes in the plant coincide with the highest fungal transcriptional activity; within the first $6 \mathrm{~h}$ following contact with the pathogen, 9,880 transcripts were upregulated and 6,261 were downregulated. From 6 to 12 hpi, 2,240 and 3,872 plant transcripts were up- and downregulated, respectively, while from 12 to $24 \mathrm{hpi}, 4,351$ transcripts were upregulated and 3,288 were downregulated. Fewer differentially expressed transcripts were observed between the mock-inoculated and 0 hpi samples, suggesting that the majority of transcriptional changes observed postinfection at later timepoints were due to responses to the fungus.

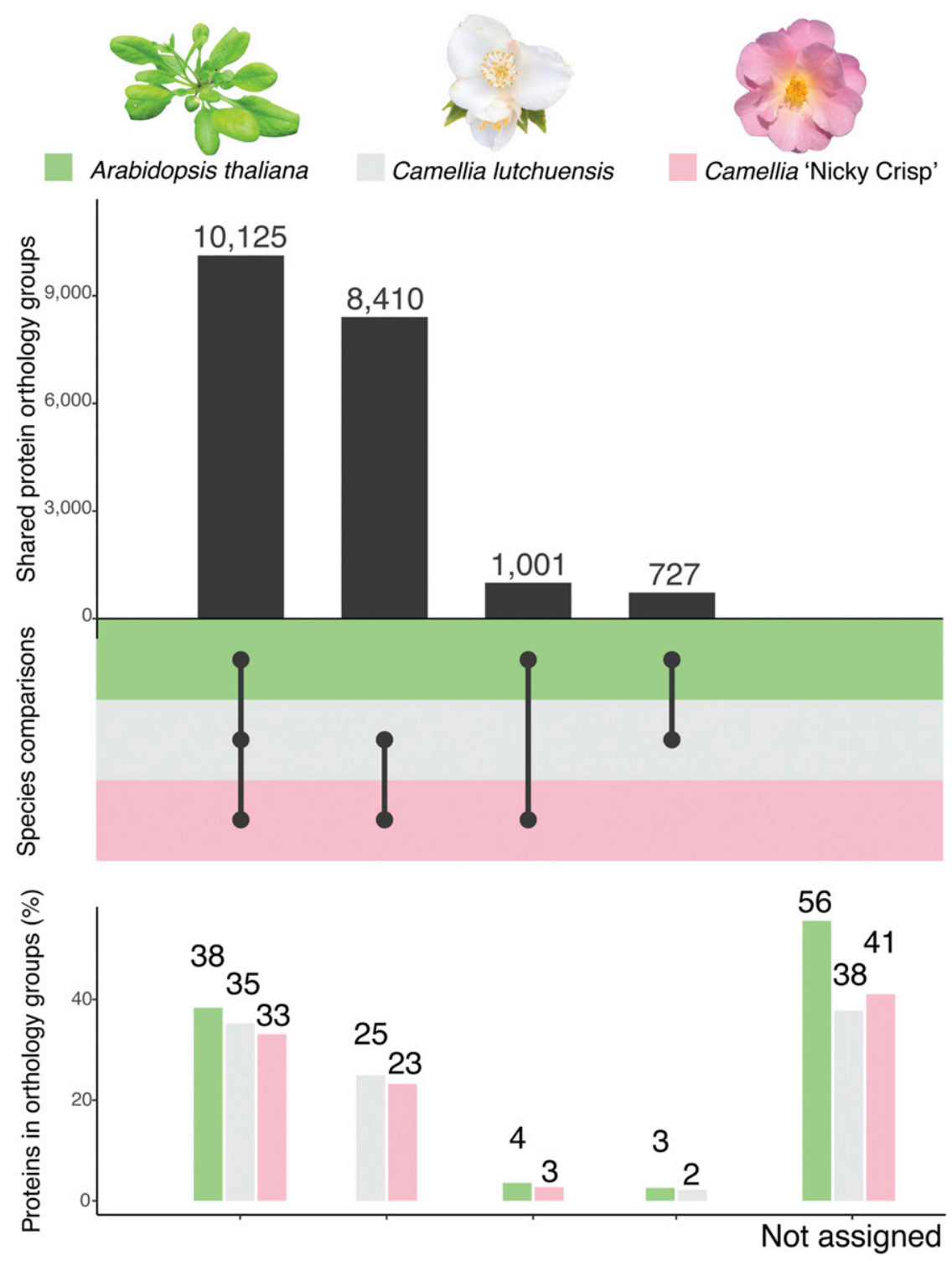

Fig. 1. Comparison of predicted Camellia proteomes with the reference proteome of Arabidopsis thaliana. Top, the number of predicted protein orthology groups. Bottom, the fraction of all protein sequences in corresponding orthology groups (the leftmost bars show proteins that were classified into orthology groups shared by all three species). 
Functional enrichment of $C$. lutchuensis expression triggered by Ciborinia camelliae ascospores.

Identifying functional effects is challenging due to the number of gene interactions involved in most biological processes. We grouped $C$. lutchuensis transcripts based on shared patterns of change in their expression levels in response to the petal blight pathogen. Fold changes in gene expression at each timepoint and treatment condition were calculated relative to 0 -hpi levels. A total of 23,083 assembled transcripts were significantly up- or downregulated during at least one of these analysis points, and 13,598 of these transcripts had functional annotations (Supplementary Table S2). Pairwise correlation coefficients for all transcript pairs were submitted to a hierarchical clustering algorithm. GO enrichment analysis was performed on the resulting dendrogram, restricted to ten clusters (Fig. 3; Supplementary Table S3). Clusters 3, 5, and 8 represent genes upregulated in response to inoculation with fungal spores. The biggest cluster (3) contains genes with the earliest responses to the fungus as well as the most significant enrichment for 'defense response' and 'protein phosphorylation' GO terms. Clusters 5 and 8 demonstrate maximum expression at 12 and $24 \mathrm{hpi}$, respectively, and are enriched for GO terms associated with secondary metabolism, such as 'secondary metabolic process', 'secondary metabolite biosynthesis process', 'oxidation-reduction process', 'phenylpropanoid metabolic process', and 'flavonoid biosynthesis process'. Clusters 6, 7, and 9 contain transcripts downregulated during the course of infection and are enriched for macromolecule biosynthesis, organ development, and responses to light and hormones. Finally, clusters $1,2,4$, and 10 , which were not specific to the fungal treatment, contain far fewer transcripts and are enriched for responses to abiotic stimuli such as 'response to red light', 'temperature compensation of the circadian clock', and 'hypotonic salinity response'. Overall, the observed functional enrichments of differentially expressed genes in resistant C. lutchuensis are consistent with typical plant resistance responses.

\section{Correlated $C$. lutchuensis transcripts that respond to Ciborinia camelliae ascospores form interaction networks.}

Similar expression dynamics for a set of genes may suggest that they are involved in the same cellular process or produce proteins that interact to form a functional complex. Such interactions tend to be evolutionarily conserved (Ho et al. 2012; Musungu et al. 2015). We therefore grouped transcripts with similar expression patterns in $C$. lutchuensis and identified their predicted orthologs in A. thaliana. The orthologs of transcripts from each predicted expression cluster form nonrandom interaction networks with significantly higher rates of interaction than expected by chance (all $P<0.001$ ) (Fig. 4). Thus, we concluded that predicted $C$. lutchuensis transcript clusters include important defense-associated molecular networks.
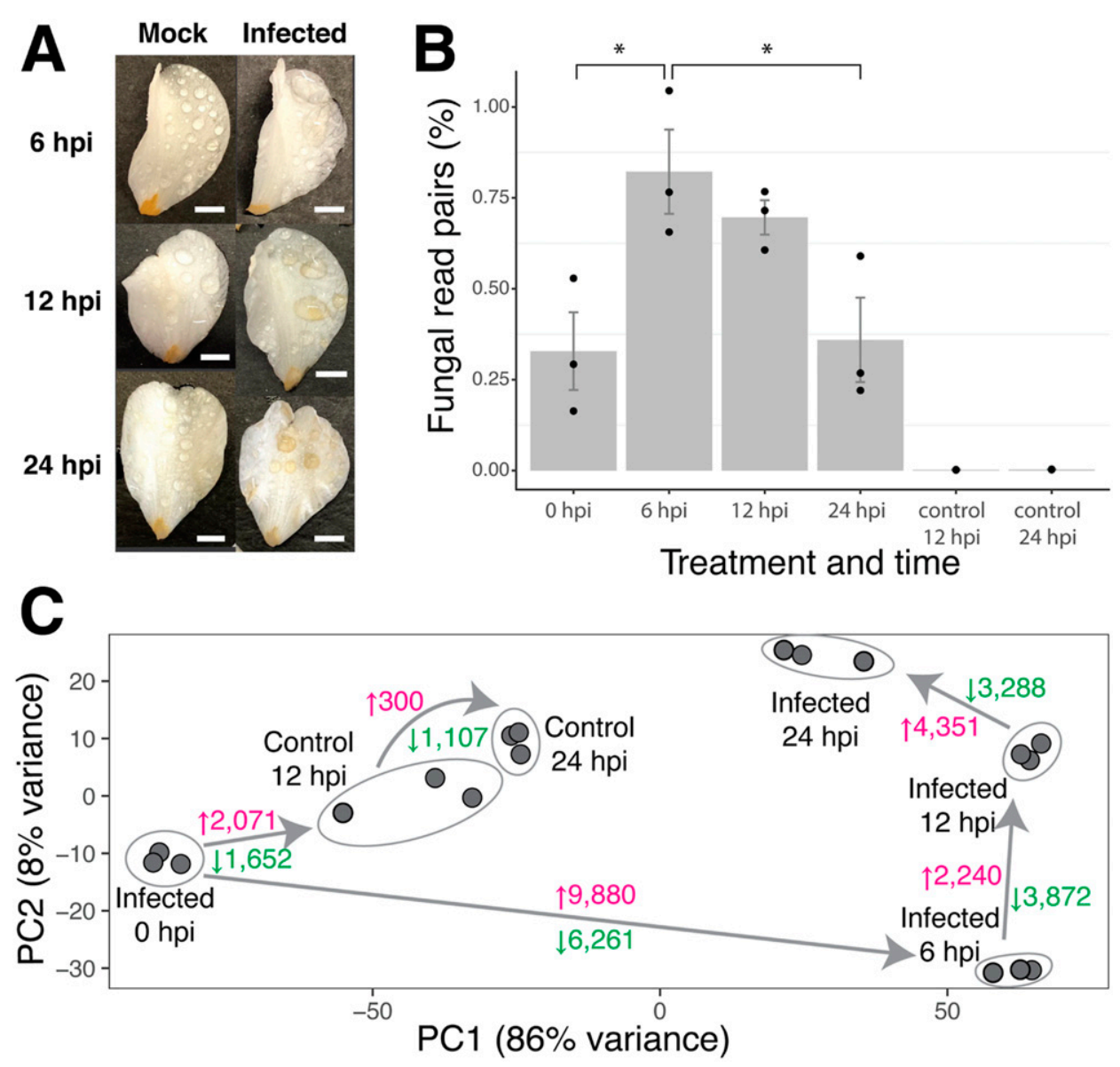

Fig. 2. Interactions between Ciborinia camelliae and resistant Camellia lutchuensis. A, Development of the resistance response in C. lutchuensis. Scale bar $=$ $0.5 \mathrm{~cm}$. B, Proportion of reads mapped to the fungal genome. Each dot represents an observed value, bars represent the average value at each timepoint and treatment, error bars represent standard error. Asterisks (*) indicate $P<0.05$ (two-tailed Student's $t$ test). C, Principal component analysis of plant read count data. Axes show first and second principal components (PC1 and PC2, respectively). Each dot represents a biological replicate, circles represent matched biological replicates, arrows indicate the time course, numbers represent upregulated (pink) or downregulated (green) transcripts. hpi $=$ hours postinoculation. 
Ciborinia camelliae-responsive interaction networks contain defense-associated hubs and key enzymes of jasmonate and phenylpropanoid biosynthesis.

Functional biological networks contain key hub elements that form multiple interactions. We identified important regulatory transcripts (hub transcripts) in three predicted networks (3, 5, and 8), which represent $C$. lutchuensis transcripts with the highest upregulation at 6, 12, and 24 hpi, respectively (Fig. 4). Each node (transcript) in these networks is represented on a scatterplot (Fig. 5; Supplementary Table S4), in which the $x$ axis shows the number of interactions that each transcript forms within the predicted network and the $y$ axis shows the $\log _{2}$ foldchange value of each transcript at 6,12 , or 24 hpi for networks 3,5 , and 8 , respectively. Transcription factors, kinases, and ubiquitin-ligases are involved in a range of cellular processes and are expected to interact with multiple gene targets. These three functional groups are enriched in transcripts with maximal upregulation at 6 versus 12 and $24 \mathrm{hpi}\left(\chi^{2}\right.$ independence test, both $P<0.01$, degrees of freedom $=3$ ), especially at highfrequency interaction hubs. Three of the transcripts that were highly upregulated at $6 \mathrm{hpi}, W R K Y 33, M P K 3$ and $M Y B 15$, are essential for disease resistance in A. thaliana (Beckers et al. 2009; Birkenbihl et al. 2012; Chezem et al. 2017).

Production of secondary metabolites plays an important role in plant resistance to various pathogens (Pusztahelyi et al. 2015). Indeed, networks corresponding to the highest expression levels at 12 and 24 hpi contain enzymes associated with secondary metabolite biosynthesis, such as 4-coumarate-CoA ligase (4CL) and cinnamate-4-hydroxylase $(\mathrm{C} 4 \mathrm{H})$, which are enzymes central to the phenylpropanoid pathway; ferulate 5hydroxylase $(\mathrm{F} 5 \mathrm{H})$, which is the rate-limiting enzyme of syringyl lignin biosynthesis; and elicitor-activated gene 3

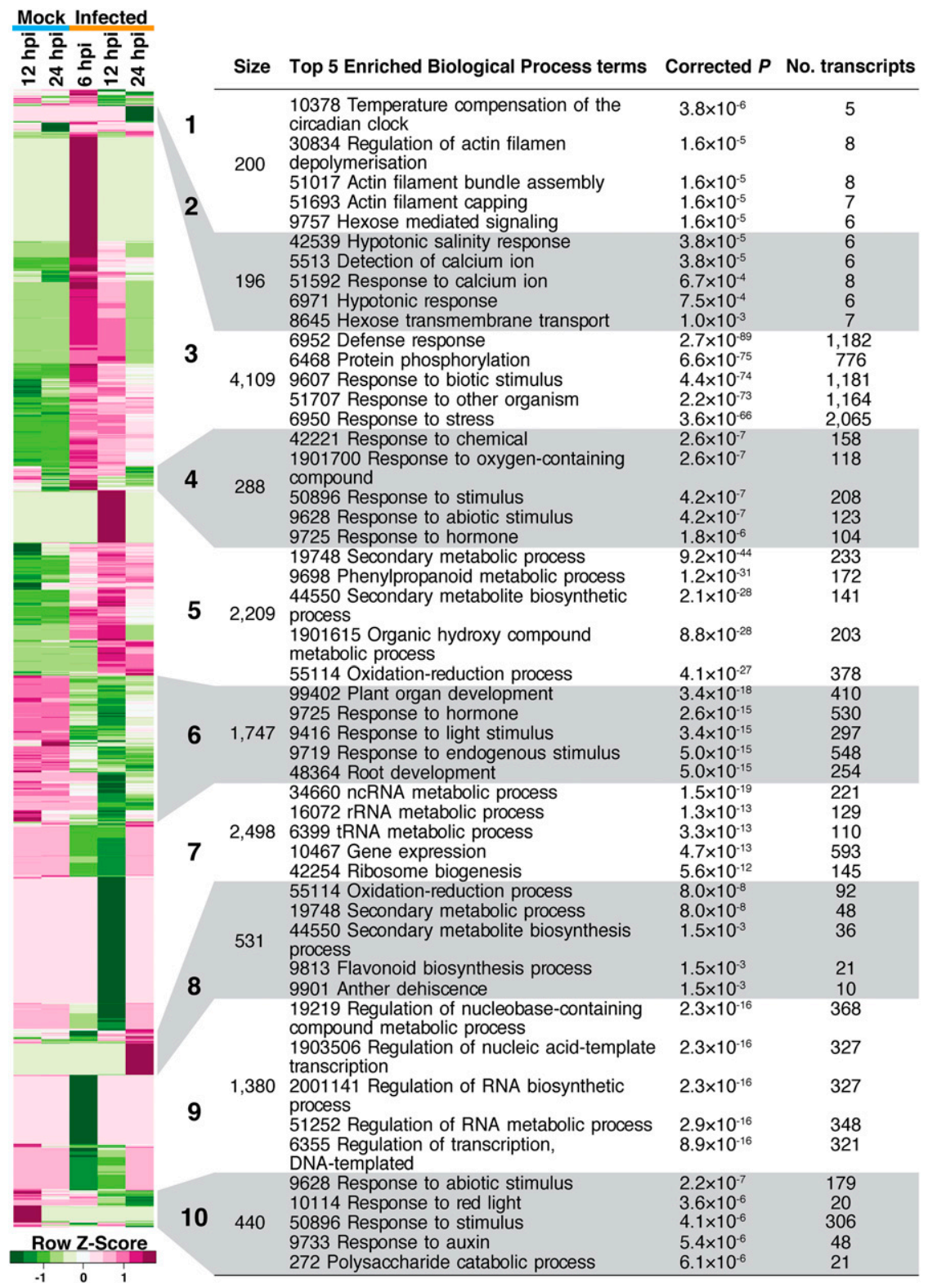

Fig. 3. Gene ontology (GO) enrichment of Camellia lutchuensis transcripts that respond to Ciborinia camelliae. Left, a heatmap showing row-scaled log ${ }_{2}$ foldchange values for 13,598 differentially expressed and annotated $C$. lutchuensis transcripts. Fold changes are presented relative to $0 \mathrm{~h}$ postinoculation (hpi). Each row corresponds to a transcript, each column to a treatment and timepoint. Pink = upregulated genes, green = downregulated. White and gray bars correspond to ten predicted transcript clusters, defined on the correlation of their expression values. The table shows the number of transcripts in each cluster, the five most significantly enriched GO biological process terms, corrected probability values, and the number of cluster transcripts that had the corresponding GO term in their annotation. 
(ELI3-2), which encodes an aryl-alcohol dehydrogenase and participates in lignin biosynthesis. The high-frequency interaction hubs with maximal upregulation at 12 and 24 hpi were genes encoding enzymes related to secondary metabolism: aldehyde dehydrogenases $(A L D H 2 B 4, A L D H 3 H 1)$, oxidoreductase BRI1-5 ENHANCED 1 (BEN1), and dihydroflavonol 4reductase $(D F R)$

Jasmonic acid (jasmonate), a plant hormone, also mediates resistance to necrotrophic pathogens (Han 2017). Transcripts involved in jasmonate metabolism, signaling, and response to jasmonate appear in all three networks (Fig. 5, triangles), including genes encoding the main enzymes of the jasmonate biosynthesis pathway: 13S-lipoxygenase $(L O X), 12$-oxophytodienoate reductases $(O P R)$, allene oxide synthase $(A O S)$, OPC-8:0 CoA ligase $1(O P C L)$, allene oxide cyclase $(A O C)$, and acyl-CoA oxidase $(A C X)$. In summary, this interactomebased analysis highlights that ascospore-treated resistant C. lutchuensis petals activated multiple interaction hubs that play a role in resistance to petal blight disease.

\section{Quantitative reverse transcription PCR (qRT-PCR)} validates the results of quantitative RNA-seq analysis.

We verified the quantitative RNA-seq results in resistant C. lutchuensis by measuring the expression levels of genes belonging to resistance-associated hubs. We tested WRKY33, $M P K 3$, and $M Y B 15$, which are the hub genes that form multiple interactions and are highly expressed at an early stage of infection in resistant plants. We also analyzed the expression of
$B A K 1, S O B I R 1$, and RIN4, which are upregulated at 6 hpi and whose involvement in $A$. thaliana immunity has been reported previously (Liebrand et al. 2014; Toruño et al. 2019), as well as $L O X, A O S, O P C L$, and $O P R$, which are involved in jasmonate biosynthesis. qRT-PCR expression data for all 10 tested genes correlated highly with the results obtained from the quantitative RNA-seq analysis, validating these key observations (all $P<$ 0.05, Pearson's correlation test) (Fig. 6A).

\section{Resistance-associated hubs have elevated expression levels at late stages of infection in susceptible Camellia 'Nicky Crisp'.}

We surveyed the expression of a subset of genes in the highly susceptible Camellia 'Nicky Crip', using qRT-PCR, to determine whether they show different expression dynamics relative to the resistant species. We analyzed six early resistance hub genes (WRKY33, MPK3, MYB15, SOBIR1, BAK1, RIN4), three genes that encode phenylpropanoid pathway enzymes $(\mathrm{C} 4 \mathrm{H}, 4 \mathrm{CL}, \mathrm{F5H})$, and four jasmonate-biosynthesis genes (LOX, AOS, OPCL, OPR). All 13 genes showed divergent expression patterns in the susceptible plants (Fig. 6B). None were significantly upregulated after the mock treatment at any timepoint, validating that their expression only responded to fungal spores. However, after fungal treatment, the transcripts had slightly elevated expression levels at $6 \mathrm{hpi}$, but this was only significant for $B A K 1$ and SOBIR1. The highest upregulation for all transcripts was seen at $24 \mathrm{hpi}$, in contrast to the rapid and strong upregulation observed at $6 \mathrm{hpi}$ in $C$. lutchuensis.
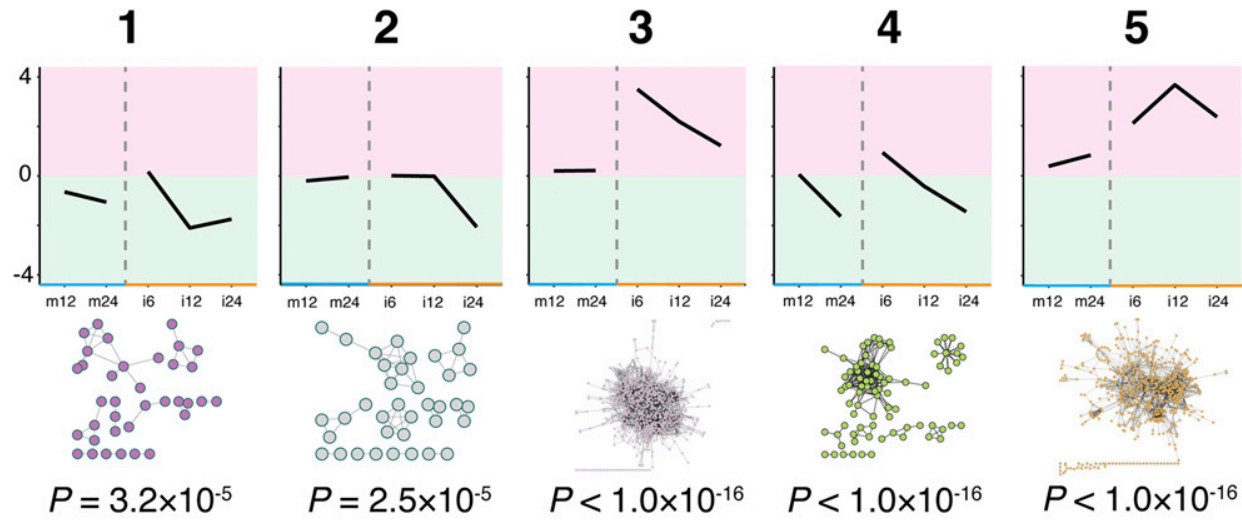

6
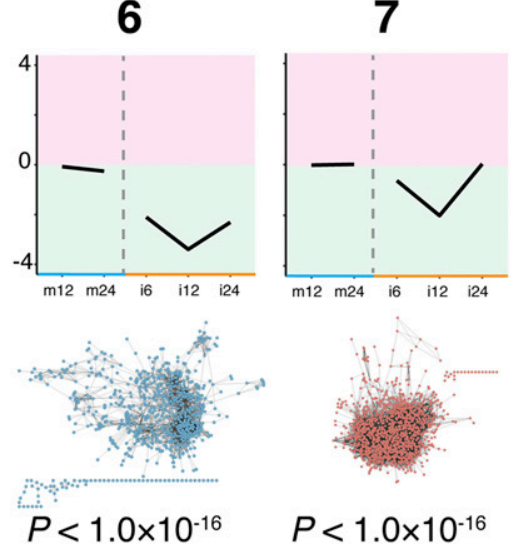

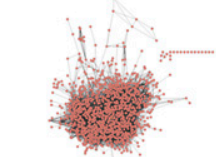

$P<1.0 \times 10^{-16}$
8

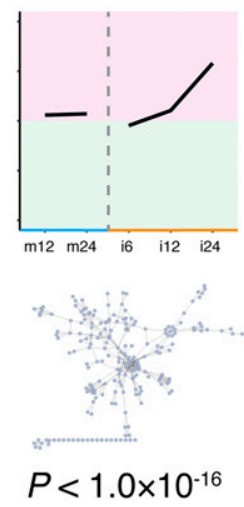

$P<1.0 \times 10^{-16}$
9
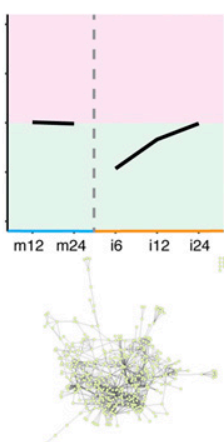

$P<1.0 \times 10^{-16}$

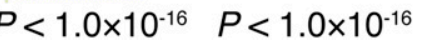

Fig. 4. Interaction networks of Arabidopsis thaliana orthologs corresponding to correlated Camellia lutchuensis transcripts that respond to Ciborinia camelliae infection. Line charts show average expression patterns of $C$. lutchuensis transcript clusters. Red background corresponds to upregulation and green to downregulation. The $y$ axes show $\log _{2}$ fold-change values, and the $x$ axes show time and treatment $(\mathrm{m} 12=$ mock $12 \mathrm{~h}$ postinoculation [hpi], $\mathrm{m} 24=\mathrm{mock} 24 \mathrm{hpi}$, i6 $=$ infected $6 \mathrm{hpi}$, i12 = infected $12 \mathrm{hpi}$, i24 = infected $24 \mathrm{hpi}$ ). Networks show the interactions between $A$. thaliana genes that are predicted orthologs of $C$. lutchuensis transcripts from indicated clusters. Probability values show the chance of observing a network with the same size and connectivity from a set of randomly sampled $A$. thaliana proteins (all $P<0.001$ ). 
Activation of resistance hubs in susceptible Camellia 'Nicky Crisp', therefore, occurred later than in the resistant C. lutchuensis.

\section{Methyl jasmonate (MeJA) treatment suppresses petal blight infection and upregulates resistance-associated hubs in susceptible Camellia 'Nicky Crisp'.}

MeJA, a volatile derivative of jasmonic acid, is widely used to protect plants against fungal diseases (Wang et al. 2015; Zhu and Tian 2012). Since the resistance-associated hubs observed in $C$. lutchuensis include jasmonate production, signaling, and jasmonate-induced responses, it was considered that jasmonate treatment may be used to induce petal blight resistance-like processes in susceptible Camellia spp. and mimic early plant defense. To test this hypothesis, we sprayed petals with the same fungal spore solution but added either MeJA or ethanol (control) (Fig. 7A and B). At 48 hpi, lesions fully covered ascospore-treated petals, while lesion sizes were significantly smaller in petals treated with MeJA $\left(P=2.9 \times 10^{-5}\right.$, two-tailed Mann-Whitney $U$ test). We assayed expression levels of the $M P K 3$ and WRKY33 genes in susceptible plant petals $6 \mathrm{~h}$ after the treatment. While the MeJA-treated petals showed higher gene expression levels than ascospore-treated ones, only the infection with ascospores combined with the hormone treatment resulted in a significant upregulation of two key defense genes (Fig. 7C). Taken together, these observations suggest that early activation of resistance-associated hubs by jasmonate likely contributes to the Camellia defense against petal blight disease.

\section{Petal blight-resistant $C$. lutchuensis shows a susceptibility-like response \\ to Ciborinia camelliae mycelial inoculum.}

Previous experiments showed the importance of the early defense activation for petal blight resistance. Since the early defense response coincided with ascospore germination and primary hyphal growth (Denton-Giles et al. 2013), we hypothesized that these initial processes may activate the early defense response and, hence, if we prevent these early events from occurring, the defense response may be delayed. Thus, we challenged susceptible and resistant Camellia petals with agar plugs containing actively growing aseptically cultivated Ciborinia camelliae mycelium. Unlike treatments with ascospores, mycelial inoculum caused infection in both susceptible and resistant Camellia petals (Supplementary Fig. S2). To further characterize the interaction between plants and fungal mycelium, we assayed the expression of MPK3 and WRKY33 genes. In accordance with previous results, ascospore-treated C. lutchuensis petals showed rapid upregulation of both resistance hubs at $6 \mathrm{hpi}$, which subsided to control levels at $24 \mathrm{hpi}$ (Fig. 8A; Supplementary Fig. S2) (both $P<0.05$, according to linear regression analysis). In contrast, the interaction of
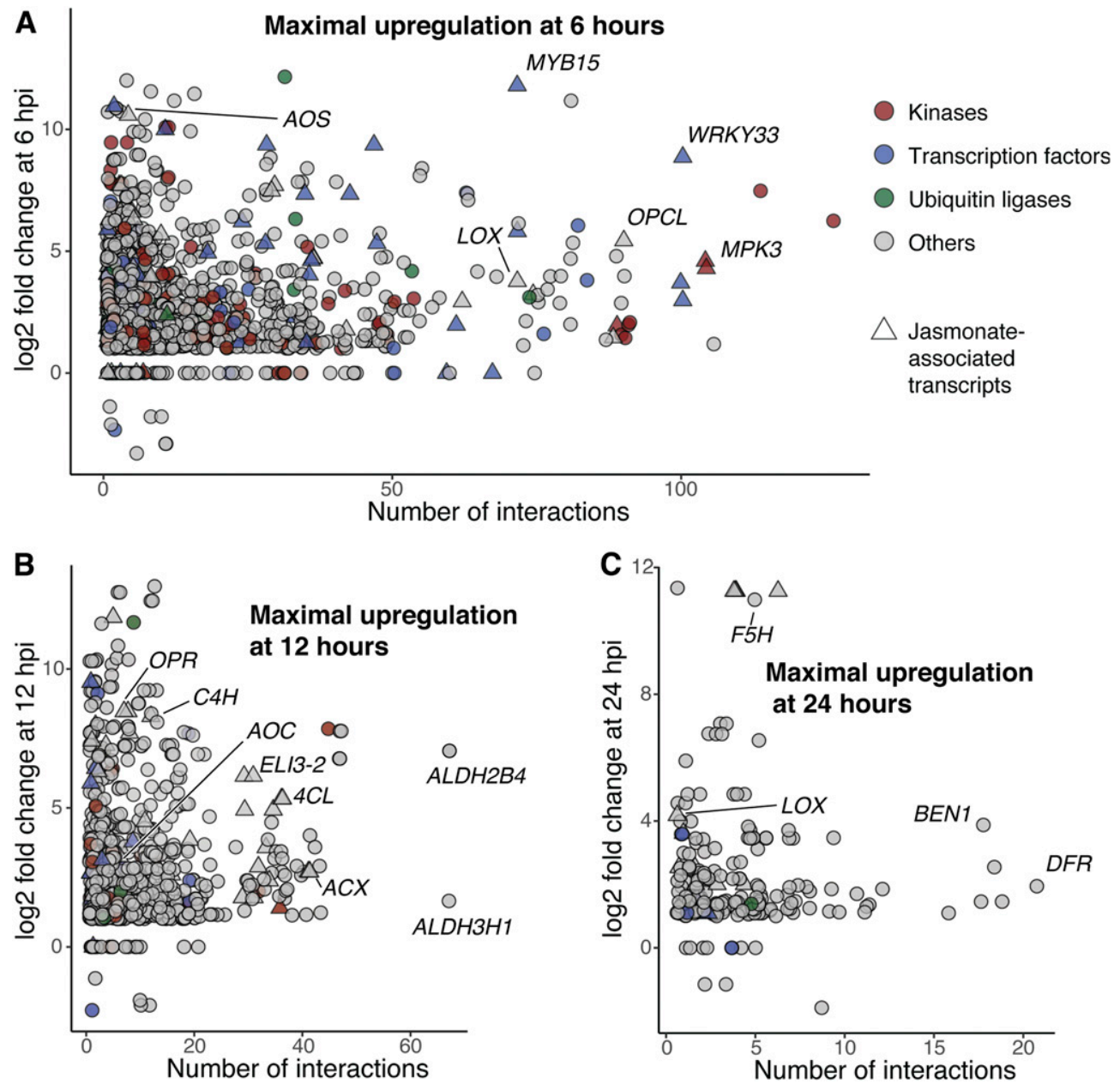

Fig. 5. Predicted interactome nodes. A, Dots represent Camellia lutchuensis transcripts from expression clusters $3, \mathbf{B}, 5$, and $\mathbf{C}, 8$. The $x$ axes show the number of interactions for the ortholog of each transcript in the corresponding Arabidopsis thaliana interaction network. The $y$ axes show $\log _{2}$ fold-change of transcription at 6, 12, or $24 \mathrm{~h}$ postinoculation for clusters 3,5, and 8, respectively. Functional groups are denoted by different colors, transcripts with GO terms that contain key word 'jasmonic acid' are shown as triangles, key genes of note that are described in the text are labeled. 
C. lutchuensis with fungal mycelium showed delayed increase in the expression of the resistance hubs, where, at $24 \mathrm{hpi}$, expression was higher than at 6 hpi (Supplementary Fig. S2; Figure 8A) (both $P<0.05$, according to linear regression analysis), reminiscent of dynamics observed for the susceptibility response. As expected, both spore and mycelial inocula induced the resistance hubs to a greater extent at 24 hpi than at 6 hpi in the susceptible hybrid (Fig. 8B) (all $P<0.05$, according to linear regression analysis).

\section{DISCUSSION}

When plant pathogens circumvent pre-existing structural and chemical defenses, plants must rely on their innate immune system, which is based on the recognition of nonself (Hahlbrock et al. 2003) and subsequent activation of defense responses. This typically results in localized necrosis (Cook et al. 2015), which can effectively stop biotrophic pathogens that require a living plant host for growth and development. However, necrotrophs explicitly exploit this response to grow on the necrotized tissue (Ciuffetti et al. 2010; Gilbert and Wolpert 2013). Nevertheless, most plants show resistance to a wide variety of necrotrophic fungi and, here, we show that for the Camellia spp.-Ciborinia camelliae interaction, the timing rather than the response itself defines the outcome of the disease process.

Global gene expression analysis identified major functional patterns of $C$. lutchuensis resistance to Ciborinia camelliae ascospores. The defense response of resistant Camellia petals followed a typical growth-defense tradeoff (Huot et al. 2014) and included two temporally segregated components, i.e., early kinase activity and signaling and, subsequently, secondary metabolite production (Hahlbrock et al. 2003). Similarly, Brassica napus resistance to $S$. sclerotiorum involved the activation of mitogen-activated protein kinase signaling cascades and resulted in the production of glucosinolates $(\mathrm{Wu}$ et al. 2016). C. lutchuensis transcript coexpression groups also had orthologous interaction networks in A. thaliana, showing that the resistance of $C$. lutchuensis to Ciborinia camelliae is typical of defense mechanisms to pathogens found in other plants.

We highlighted the main regulatory pathways of the defense response, using an interactome-based approach. Key genes were upregulated in resistant $C$. lutchuensis petals after ascospore treatment, with the strongest interaction hubs containing orthologs of known resistance- and stress-associated genes, such as WRKY33 (Birkenbihl et al. 2012), MPK3 (Mao et al. 2011), and MYB15 (Höll et al. 2013). The WRKY33 transcription factor and $M P K 3$ kinase are involved in a common defense regulatory loop in $A$. thaliana that contributes to systemic acquired resistance (Wang et al. 2018b). The MYB15 transcription factor is a positive regulator of the phenylpropanoid pathway (Chezem et al. 2017). We also observed enriched expression of phenylpropanoid pathway genes, which may contribute to Ciborinia camelliae resistance via the production of compounds with direct antifungal activity (König et al. 2014) and as precursors of lignin monomers that reinforce plant cell walls (Wang et al. 2013). Oxylipin pathway genes involved in the biosynthesis of jasmonic acid, a plant hormone that mediates resistance against necrotrophic pathogens (Han 2017), were upregulated in several interaction hubs. Nevertheless, all three
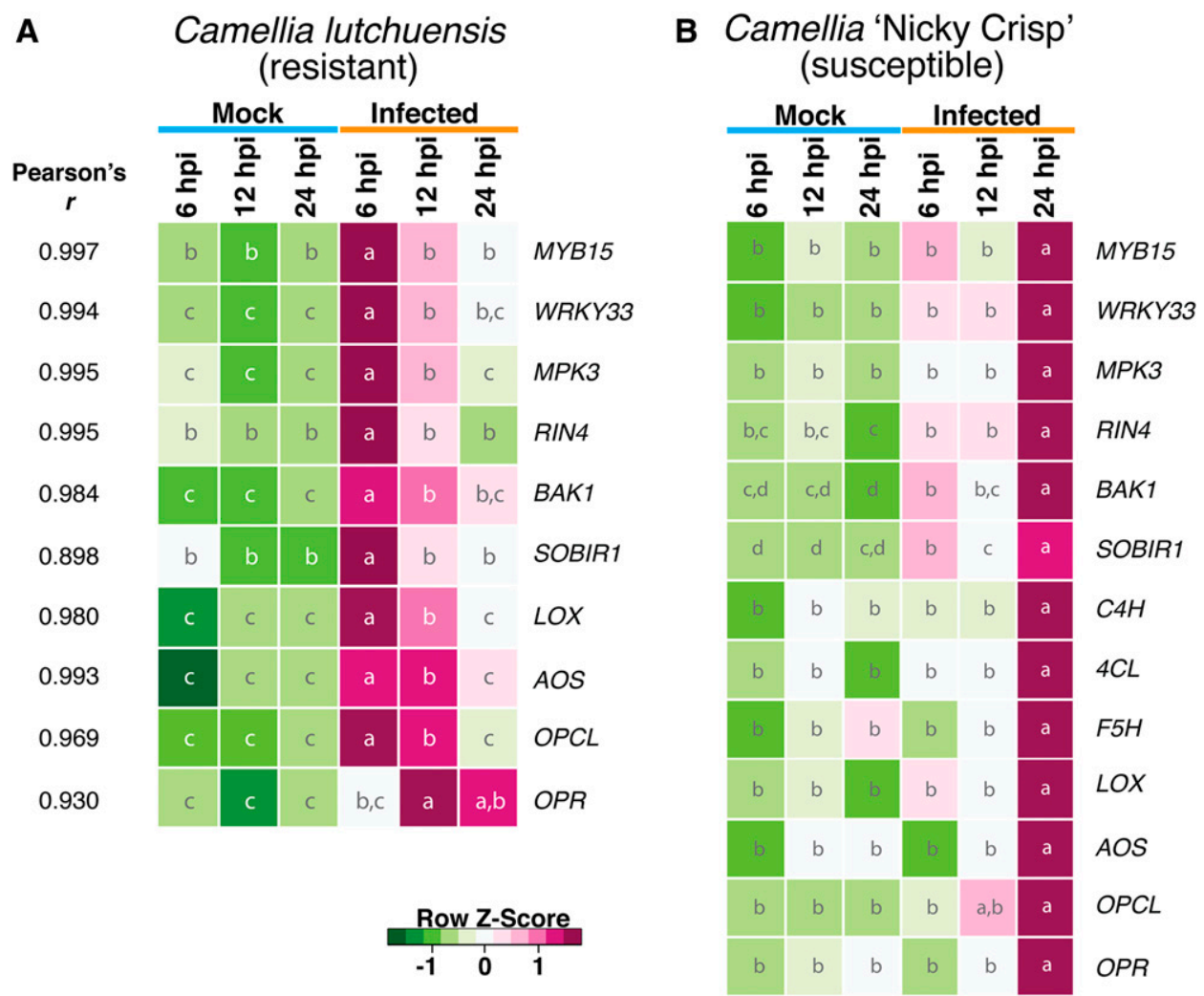

Fig. 6. Quantitative reverse transcription PCR (qRT-PCR) analysis of selected transcripts. A, Row-scaled heatmaps represent expression levels in Camellia lutchuensis and B, Camellia cultivar Nicky Crisp. Each row represents a gene transcript, each column a treatment and timepoint. Pink $=$ upregulated genes, green $=$ downregulated. Relative expression levels were measured against two reference transcripts (PTB1 and EF1- $\alpha$ for C. lutchuensis and PTB1 and Tubulin $\alpha-3$ for Camellia 'Nicky Crisp'). Expression levels are presented as the mean of three biological replicates. Data were analyzed with one-way analysis of variance and Tukey's honest significant difference tests separately for each transcript. The same letter in two heatmap cells within a row indicates that expression levels did not differ significantly between those two conditions $(P<0.05)$. For $C$. lutchuensis, correlations between RNA-seq and qRT-PCR expression values (left column in A) were tested with Pearson's correlation coefficient $($ all $P<0.05)$. hpi $=$ h postinoculation. 
defense pathways tested were significantly upregulated during the susceptible interaction as well, albeit at a later stage of the infection.

The striking correlation between timing of the response and the outcome of the disease-i.e., resistance or susceptibility-led us to hypothesize that the response timing is important for both resistance and susceptibility. To test this, we induced an early response in the susceptible hybrid and manipulated the infection in the resistant species to delay the response, expecting to see opposite outcomes of the infection. Indeed, the exogenous application of MeJA, which led to the upregulation of two important resistance hubs, WRKY33 and $M P K 3$, within the first $6 \mathrm{~h}$ of infection, strongly reduced lesion sizes in susceptible petals. This further verifies that early defense activation contributes to petal blight resistance. Remarkably, the interaction of resistant C. lutchuensis with actively growing Ciborinia camelliae mycelium but not ascospores delayed elevated expression of the resistance hubs and resulted in a susceptibility interaction. This also suggests that early events, such as spore germination or primary hyphal growth, are critical for rapid induction of the resistance response in the resistant species. Further research is required to determine the contribution of the detected molecular defense pathways to petal blight resistance and whether the production of jasmonates occurs as part of the defense response or even plays a role as primary defense activator.

A timing-based defense interaction agrees with previously reported developmental dynamics of the petal blight pathogen. A distinctive feature of the disease is its rapid spread of infection; while other fungal infections develop over days or weeks, a single Ciborinia camelliae lesion spreads over the whole petal within $48 \mathrm{~h}$ (Denton-Giles et al. 2013). As shown by the RNAseq results, the proportion of fungal RNA in infected samples doubled in the first $6 \mathrm{~h}$ of the infection. The transcriptional response of resistant plants was similarly quick, with maximal activity at $6 \mathrm{hpi}$. This accords with microscopic observations showing that the resistance response against ascospores occurs within the first $12 \mathrm{~h}$ of interaction, during the asymptomatic phase, with localized plant cell death, papillae formation, and accumulation of reactive oxygen species (Denton-Giles et al. 2013). Plant resistance thus appears to be most effective during the biotrophy-like asymptomatic growth stage of the fungus (Denton-Giles et al. 2013) — the initial phase found even in 'classic' necrotrophs such as $S$. sclerotiorum (Kabbage et al. 2015) and B. cinerea (Veloso and van Kan 2018). Similar to our observations, necrotrophic Macrophomina phaseolina infection of a resistant plant line of Seasamium indicum showed a prompt host response to the pathogen at the initial biotrophy-like stages of the interaction (Chowdhury et al. 2017).

It is still unclear what the main factors are that regulate the timing of Camellia defense. Susceptible Camellia species may lack the immune receptors needed to detect early Ciborinia camelliae activity, hence preventing prompt recognition. The pathogen might then activate host defense pathways in its favor using damage-associated molecular patterns released from cellwall destruction (Denoux et al. 2008) or secreted fungal carbohydrate-active enzymes in a similar way to $B$. cinerea (Zhu et al. 2017). Alternatively, the secretome of Ciborinia camelliae produced during the early biotrophy-like stage may contain
A

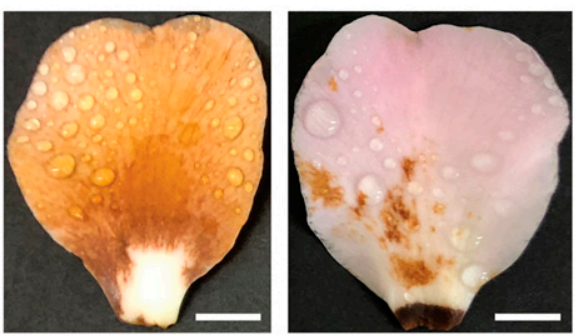

B

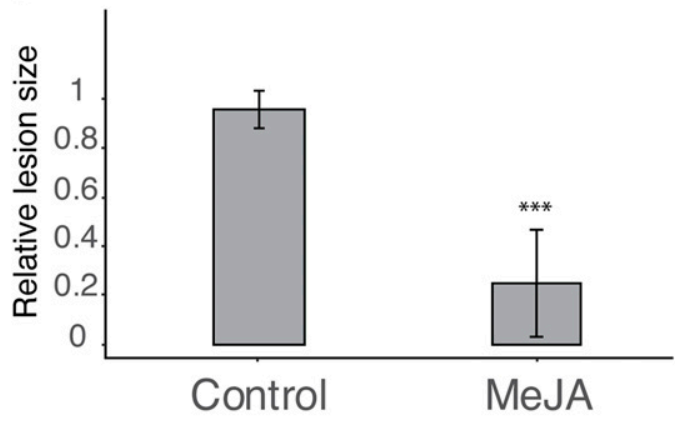

Treatment
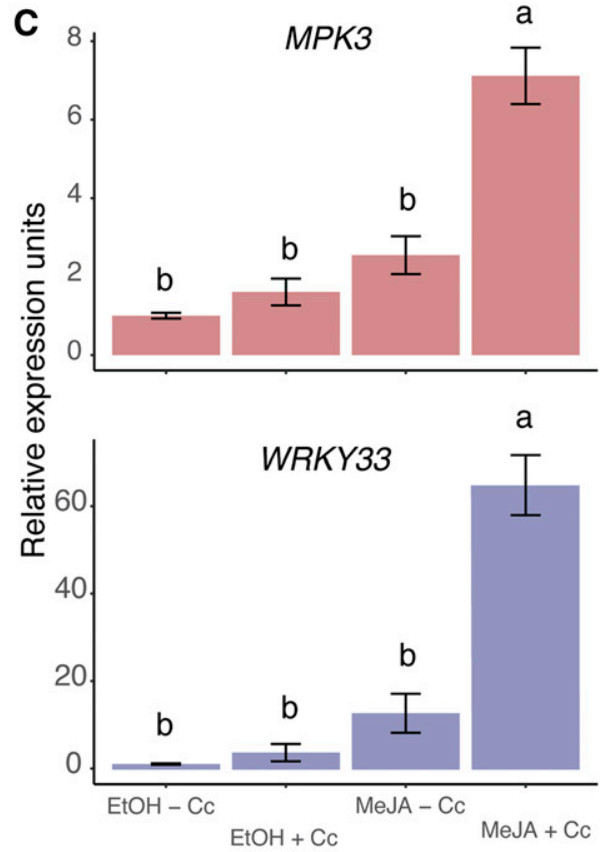

Treatment

Fig. 7. Effects of methyl jasmonate (MeJA) treatment on petal blight infection in susceptible Camellia cultivar Nicky Crisp. A, The petals on the left were treated with Ciborinia camelliae spores in $1 \%$ ethanol solution (left) or with fungal spores in a $10 \mathrm{mM}$ MeJA in $1 \%$ ethanol solution (right) and were incubated for $48 \mathrm{~h}$. Scale bar $=1 \mathrm{~cm}$. B, The bar chart shows the relative lesion sizes on petals at $48 \mathrm{~h}$ postinoculation (hpi). Bars indicate mean relative lesion areas as a proportion of the whole petal, error bars indicate the standard deviation calculated from 12 biological replicates. Asterisks $(* * *)$ indicate $P<0.001$ (two-tailed Mann-Whitney $U$ test). C, Bar charts show the effect of MeJA and spore treatment on the expression levels of WRKY33 and $M P K 3$ transcripts at 6 hpi. The $x$ axes show the treatments applied: $\mathrm{EtOH}-\mathrm{Cc}=1 \%$ ethanol only, EtOH $+\mathrm{Cc}=$ Ciborinia camelliae spores in $1 \%$ ethanol solution, $\mathrm{MeJA}-\mathrm{Cc}=10 \mathrm{mM} \mathrm{MeJA}$ in $1 \%$ ethanol solution, and MeJA + Cc $=10 \mathrm{mM}$ MeJA in $1 \%$ ethanol solution containing Ciborinia camelliae spores. Relative expression levels were measured against two reference transcripts (PTB1 and Tubulin $\alpha-3)$ and were normalized to the average value of the $1 \%$ ethanol-treated control samples. Bars show mean expression values; error bars show the standard error calculated from three biological replicates. The data were analyzed with a one-way analysis of variance and Tukey's honest significant difference test separately for each tested transcript. The same letter above two bars indicates that expression levels did not differ significantly between those two conditions $(P<0.05)$. 
compounds that suppress the immune response of susceptible plants, such as effector proteins (Zhu et al. 2013) or oxalic acid (Williams et al. 2011), as produced by the related pathogen S. sclerotiorum.

Our observations are in agreement with the Invasion Model (Cook et al. 2015), which states that plants generally interact with biotrophs and necrotrophs using the same molecular pathways but with different outcomes, inhibition of biotrophic infection and acceleration of necrotrophic infection. Figure 9 illustrates this pattern for the Camellia spp.-Ciborinia camelliae interaction; both resistant and susceptible plants trigger the same defense pathways. Early defense activation during the biotrophylike stage of fungal development, possibly induced by spore germination or primary hyphal growth, leads to host resistance; activation of the same plant defense pathways when the fungus reaches its necrotrophic stage instead leads to susceptibility. This conclusion is based on the comparative analysis of just one resistant and one susceptible host, and further work with other Camellia cultivars is needed to confirm the model.

Whether infection is more a function of the pathogen or of the host plant is important for effective treatments. For rapid infection by necrotrophic pathogens, the timing of the host response may be the more crucial factor for resistance than the nature of the defense itself and should, therefore, be considered in the design of disease-control strategies.

\section{MATERIALS AND METHODS}

Plant and fungal materials and pathogenicity assays.

Twelve C. lutchuensis Ito (Wairere Nursery) and 12 Camellia 'Nicky Crisp' $(C$. japonica $\times C$. pitardii var. pitardii) (Kilmarnock Nurseries) shrubs were grown in the Massey

\section{A Camellia lutchuensis resistant}
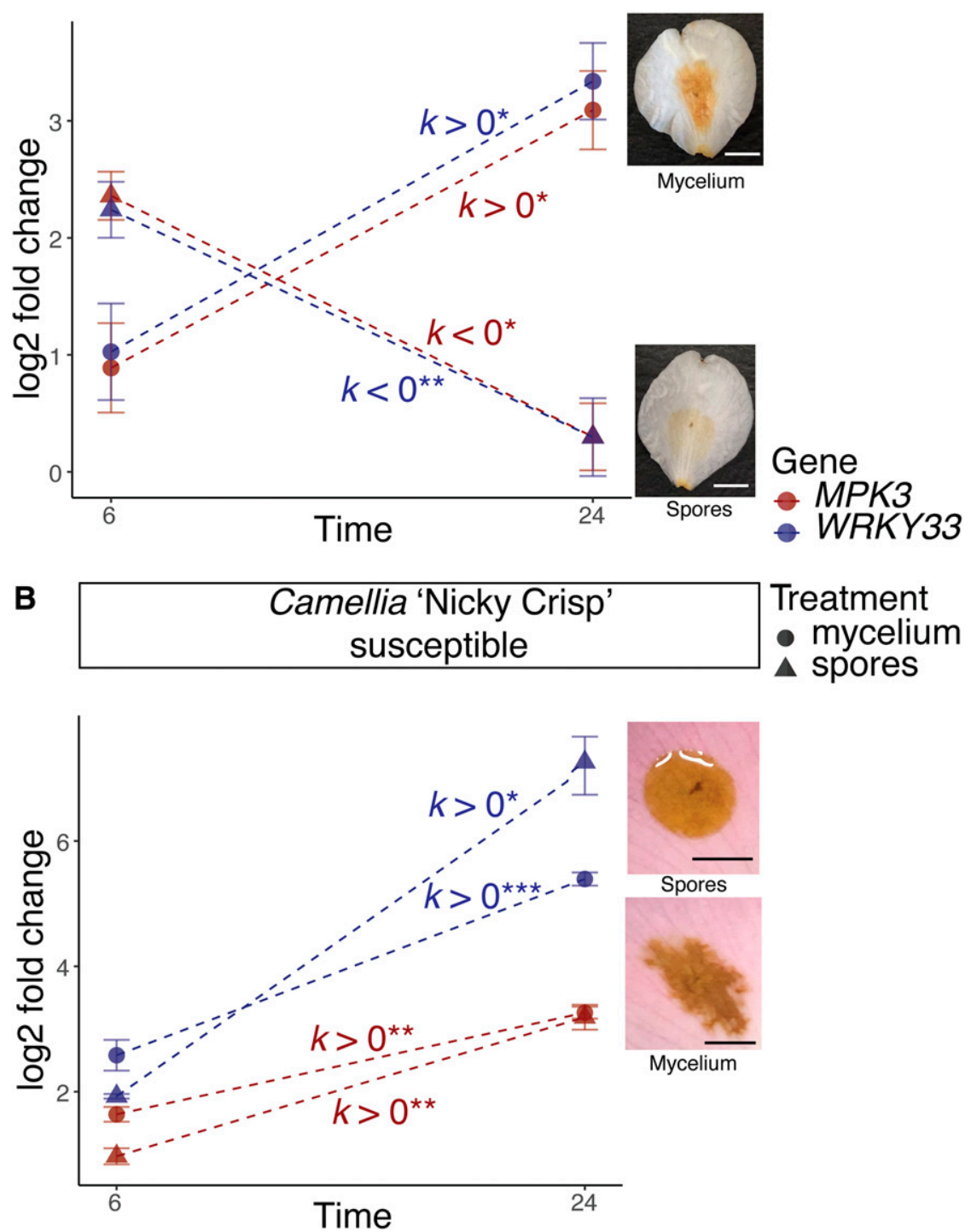

Fig. 8. Activity of Camellia resistance hubs in response to inoculation with ascospores and mycelium of Ciborinia camelliae. Graphs show the expression activity of resistance hubs in $\mathbf{A}$, Camellia lutchuensis and $\mathbf{B}$, Camellia cultivar Nicky Crisp. The $x$ axes show time and the $y$ axes $\log _{2}$ fold change. Relative expression levels were measured against two reference transcripts and were normalized to the average value of expression in petals inoculated with sterile agar plugs or drops of sterile water. Red corresponds to MPK3 gene and blue to WRKY33; shapes represent the type of fungal inoculum; bars show standard error from three biological replicates; $k$ is a slope of fitted regression line with the asterisks indicating corresponding $P$ values: One asterisk $(*) P<0.05$, two $(* *) P<$ 0.01 , and three (***) $P<0.001$. The photographs show infected petals at $24 \mathrm{~h}$ postinoculation. The slight brown color on ascospore-infected $C$. lutchuensis petals is a result of the resistance response. Scale bar $=0.5 \mathrm{~cm}$. 
University Plant Growth Unit glasshouse $\left(40^{\circ} 22^{\prime} 41.22\right.$ 's, $\left.175^{\circ} 36^{\prime} 48.73^{\prime \prime} \mathrm{E}\right)$ with air inlets covered with 30 -mm-thick polyester wadding to limit the entry of airborne ascospores of Ciborinia camelliae. The temperature in the glasshouse was maintained between 1 and $20^{\circ} \mathrm{C}$, mimicking natural conditions for these species.

Ciborinia camelliae Kohn apothecia were collected at Massey University Arboretum (40²2'58.7'S 175³7'11.6”E) between July and September. The apothecia were placed in $25-\mathrm{ml}$ glass tubes in an inverted position with a damp tissue on the top of the tube and were kept for 2 days at room temperature to release ascospores. Released Ciborinia camelliae ascospores were collected by washing the tubes with $1 \mathrm{ml}$ of sterile distilled water and spore concentrations were measured using a Neubauer-improved hemocytometer.

To infect Camellia petals with Ciborinia camelliae under controlled conditions, individual petals from fully open flowers were collected and were placed on damp tissue paper in plastic trays $\left(640 \mathrm{~cm}^{2}\right)$ and $20 \mathrm{ml}$ of ascospore solution (approximately $1.5 \times 10^{6}$ ascospores per milliliter) were sprayed on petals with an estimated final coverage of $4.5 \times 10^{4}$ to $5 \times 10^{4}$ ascospores per square centimeter. Control petals were sprayed with the same volume of sterile milliQ water. For the MeJA treatments, the required amount of $1 \mathrm{M}$ MeJA (Sigma) stock in ethanol was added to the spore solution before spraying to obtain a final concentration of $1 \%(\mathrm{vol} / \mathrm{vol})$ ethanol and $10 \mathrm{mM}$ MeJA. A control solution consisted of ethanol at a final concentration of $1 \%$ (vol/vol). For fungal mycelium inoculation, Ciborinia camelliae strain ICMP 19812 (International Collection of Microorganisms from Plants) was grown on Difco potato dextrose agar at $20^{\circ} \mathrm{C}$. Agar plugs $(0.5 \mathrm{~cm}$ diameter $)$ were obtained from the edge of actively growing 1-week-old colonies and infection was performed according to the protocol described for

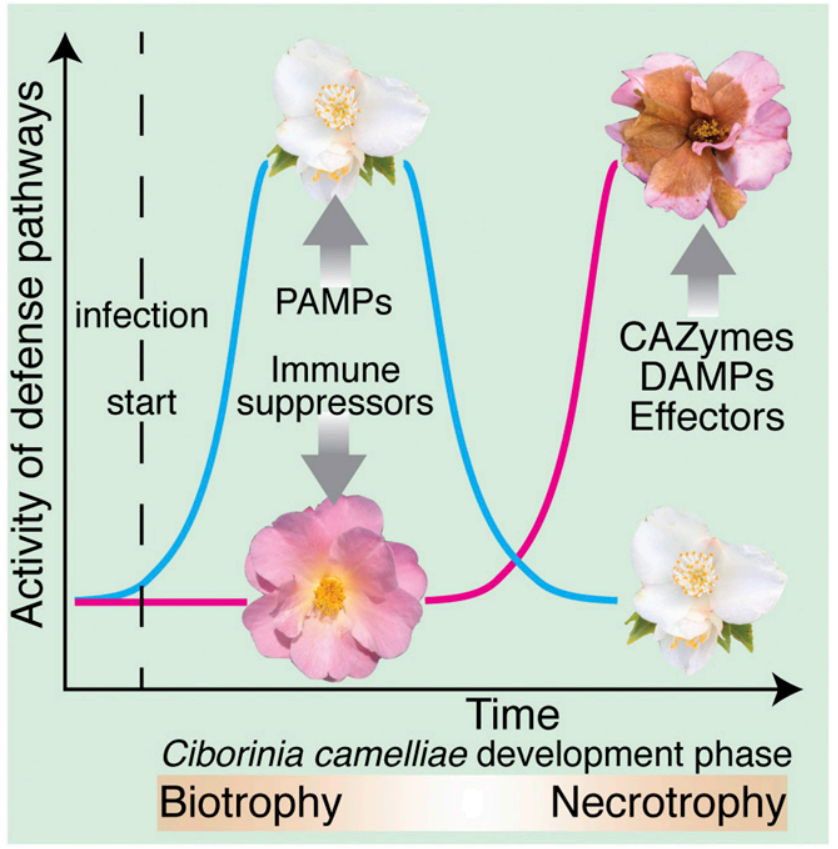

Fig. 9. Putative model of plant-fungus interaction for Camellia petal blight disease. The line chart shows the transcriptional activity of tested defense pathways in resistant Camellia lutchuensis (blue) and susceptible Camellia cultivar Nicky Crisp (red). Gray arrows show fungal molecules that can modulate plant defense pathways. The orange bar (bottom) shows the development phases of the petal blight pathogen. PAMPs = pathogen-associated molecular patterns, CAZymes = carbohydrate-active enzymes, and DAMPs = damage-associated molecular patterns.
B. cinerea (Ingle and Roden 2014). Trays with infected petals were covered with plastic wrap and were kept in a Biosyn Series 6000 controlled plant growth chamber (Contherm Scientific) at $21^{\circ} \mathrm{C}, 12$-h light and 12 -h dark period and $180 \mu \mathrm{mol}$ $\mathrm{m}^{-2} \mathrm{~s}^{-1}$ light intensity.

\section{RNA extraction and transcriptome sequencing.}

About $500 \mathrm{mg}$ of Camellia petal tissue (nine petals of C. lutchuensis or one petal of Camellia 'Nicky Crisp') was used for RNA extraction using a Zymo-Research Quick-RNA Miniprep kit (Zymo Research) according to manufacturer protocol. The genomic DNA digestion step was performed with recombinant DNase I (Roche Applied Science). RNA quality and quantity were checked with a NanoDrop spectrophotometer (Thermo Fisher) and Agilent 2100 Bioanalyzer with an RNA 6000 Nano kit (Agilent Technologies).

For reference transcriptome sequencing, one infected and one uninfected sample were collected at $48 \mathrm{hpi}$ for C. lutchuensis and Camellia 'Nicky Crisp'. Library preparation and RNA sequencing were performed by New Zealand Genomics Limited on an Illumina HiSeq 2000 (Illumina). The paired-end reads $(2 \times 100 \mathrm{bp})$ were processed and qualityfiltered using Cutadapt v1.14 (Martin 2011) and SolexaQA++ v3.1.7.1 (Cox et al. 2010) with a Phred quality score of $20(99 \%$ base call accuracy).

Transcriptome assembly and annotation. Fungal reads from infected samples were mapped to the reference Ciborinia camelliae genome (GenBank GCA_001247705.1) using TopHat v2.1.1 and were assembled with Cufflinks v2.2.1 (Trapnell et al. 2012), with default parameters, and unmapped reads were used to assemble de novo plant transcriptomes using Trinity v2.2.0 (Haas et al. 2013), with default parameters. Additional sequences from de novo infected petal transcriptomes were assigned to a fungal origin if those sequences aligned to $S$. sclerotiorum or B. cinerea genomes (GenBank GCA_000146945.2 and GCA_000143535.1, respectively) with E-values of $1 \times 10^{-30}$ or lower. To create reference petal transcriptomes, transcripts assembled from infected and uninfected plant samples were combined and redundant transcripts were removed, using CD-HIT-EST v4.6.4 (Li and Godzik 2006), with $90 \%$ similarity cut-off. Assembly completeness was tested with BUSCO v3 using the 'odb9' plant single-copy orthologs dataset (Simão et al. 2015).

For each transcript, best matching sequences were found in the NCBI nucleotide database using blastn and in UniProtKB/Swiss-Prot using blastx algorithms (Altschul et al. 1990) with E-value cut-off values of $1 \times 10^{-5}$. Blast2GO v3.3 (Conesa et al. 2005) was used, with default parameters, to assign GO terms (Ashburner et al. 2000), based on the best Swiss-Prot matches. TransDecoder v5 was used to predict plant proteomes from the assembled transcriptomes and Swiss-Prot search results. Orthology relationships between two predicted Camellia proteomes and the reference proteome of A. thaliana (cv. Columbia) (UniProt UP000006548) were investigated with Proteinortho v5.16b.

\section{Differential expression analysis.}

For the quantitative RNA-seq analysis, the Illumina HiSeq 2500 (Illumina, USA) platform was used to generate $2 \times 125 \mathrm{bp}$ paired-end reads (New Zealand Genomics Limited). Three biological replicates consisting of nine $C$. lutchuensis petals were collected at 6,12 , and $24 \mathrm{~h}$ after infection and at 12 and $24 \mathrm{~h}$ after mock treatment (18 samples in total). RNA extraction and read processing was performed as for the reference transcriptome assembly described above. High-quality reads from each sample were mapped onto the combined $C$. lutchuensis and Ciborinia camelliae reference transcriptomes using Bowtie 
v2.3.0 (Langmead and Salzberg 2012), and read counts were obtained from the resulting SAM files with the Perl mapcount script. Resulting count data were imported into the R software environment ( $\mathrm{R}$ Development Core Team 2017), and the DESeq2 package v1.17.18 (Love et al. 2014) was used for differential expression and PCA. A transcript was considered to be differentially expressed between two compared conditions if the $\log _{2}$ value of calculated fold change was less than -1 or greater than 1 and the adjusted $P$ value was less than 0.01 . Heatmaps were created using $\mathrm{R}$ package gplots v3.0.1.1 (Warnes et al. 2019).

\section{Prediction of expression clusters and GO-enrichment analysis.}

Pearson's correlation was calculated in $\mathrm{R}$ for the $\log _{2}$ foldchange values of each differentially expressed transcript pair; the distance between two transcripts was defined as 1 minus the correlation coefficient. The resulting distance matrix was used as input to the hclust() function in $\mathrm{R}$, which was used with default parameters to construct a clustering tree. The resulting tree was cut into 10 clusters using the cuttree() function. For each predicted transcript cluster, significantly overrepresented GO terms were found with the BinGO plug-in (Maere et al. 2005) in Cytoscape v3.6.1 (Shannon et al. 2003). The whole assembled plant transcriptome was used as a background transcript set for each enrichment analysis. Corrected enrichment $P$ values were calculated using the BinGO plug-in by means of the hypergeometric test with Bonferroni multiple testing correction.

\section{Orthology-based interactome analysis.}

Based on the protein-orthology analysis results described above, orthologous A. thaliana loci were assigned to C. lutchuensis differentially expressed transcripts. The list of corresponding TAIR (The Arabidopsis Information Resource) accession numbers was submitted to the STRING database (Szklarczyk et al. 2017) to search for interactions. 'Experiments', 'Databases', and 'Coexpression' were chosen as active interaction sources, and the minimum required interaction score was set to 0.4 (medium confidence) by default in STRING. Resulting networks were visualized and analyzed using Cytoscape v3.6.1 (Shannon et al. 2003) and the igraph R package v1.2.2 (Csárdi and Nepusz 2006). Network $P$ values were calculated in STRING and showed the probability of observing a network with the same connectivity if it was built from the same number of randomly sampled A. thaliana proteins. In addition, three random networks with sizes comparable to our networks were constructed to illustrate that their $P$ values were higher than the significance level of 0.05 . Numbers of observed interactions (graph edges) in random networks were almost equal to the expected number of interactions. The full description of predicted networks is shown in Supplementary Table S5.

\section{qRT-PCR analysis.}

cDNA synthesis was undertaken on $1 \mu \mathrm{g}$ of total RNA using Transcriptor first-strand cDNA synthesis kit with anchoredoligo $(\mathrm{dT})_{18}$ primers (Roche) per the manufacturer protocol. qPCR was performed on a LightCycler 480 Instrument II (Roche) using LightCycler 480 SYBR green I master mix (Roche) and transcript-specific primers (Supplementary Table S6). Raw data were collected from three technical and three biological replicates for each observation. Quantification cycle and primer efficiency values were calculated using LinRegPCR v2015.0 (Ruijter et al. 2009). Two reference control transcripts were chosen for each plant from the studies on Camellia sinensis (L.) O. Kuntze (Hao et al. 2014) and were then tested for stable expression at all timepoints and treatments. Eukaryotic elongation factor-1 alpha $(E F 1 \alpha)$ and polypyrimidine tractbinding protein $1(P T B 1)$ genes were used for $C$. lutchuensis and PTB1 and Tubulin alpha-3 (Tub $\alpha-3)$ for Camellia 'Nicky Crisp'. Transcript-expression fold changes were calculated relatively to their average expression level in corresponding control samples.

\section{Lesion size measurement.}

Lesion size measurements were conducted using Image J $\mathrm{v}$ 2.0.0 (Schindelin et al. 2012) as described previously (DentonGiles et al. 2013). Relative lesion size was calculated in relation to the whole petal size.

\section{Statistical analysis.}

Statistical analyses were performed using $\mathrm{R}$ base functions. Tukey's honestly significant difference test was performed with the R package agricolae v1.3 (Mendiburu and Simon 2015).

\section{ACKNOWLEDGMENTS}

We thank C. Mesarich from the School of Agriculture and Environment, Massey University, for valuable comments on the manuscript.

\section{AUTHOR-RECOMMENDED INTERNET RESOURCES}

Perl mapcount script: http://mpcox.github.io/mapcount TransDecoder v5: https://github.com/TransDecoder/TransDecoder

\section{LITERATURE CITED}

Altschul, S. F., Gish, W., Miller, W., Myers, E. W., and Lipman, D. J. 1990. Basic local alignment search tool. J. Mol. Biol. 215:403-410.

Ashburner, M., Ball, C. A., Blake, J. A., Botstein, D., Butler, H., Cherry, J. M., Davis, A. P., Dolinski, K., Dwight, S. S., Eppig, J. T., Harris, M. A., Hill, D. P., Issel-Tarver, L., Kasarskis, A., Lewis, S., Matese, J. C., Richardson, J. E., Ringwald, M., Rubin, G. M., and Sherlock, G. 2000. Gene ontology: Tool for the unification of biology. Nat. Genet. 25:25-29.

Beckers, G. J. M., Jaskiewicz, M., Liu, Y., Underwood, W. R., He, S. Y., Zhang, S., and Conrath, U. 2009. Mitogen-activated protein kinases 3 and 6 are required for full priming of stress responses in Arabidopsis thaliana. Plant Cell 21:944-953.

Birkenbihl, R. P., Diezel, C., and Somssich, I. E. 2012. Arabidopsis WRKY33 is a key transcriptional regulator of hormonal and metabolic responses toward Botrytis cinerea infection. Plant Physiol. 159: 266-285.

Chezem, W. R., Memon, A., Li, F.-S., Weng, J.-K., and Clay, N. K. 2017. SG2-type R2R3-MYB transcription factor MYB15 controls defenseinduced lignification and basal immunity in Arabidopsis. Plant Cell 29: 1907-1926.

Chowdhury, S., Basu, A., and Kundu, S. 2017. Biotrophy-necrotrophy switch in pathogen evoke differential response in resistant and susceptible sesame involving multiple signaling pathways at different phases. Sci. Rep. 7:17251

Ciuffetti, L. M., Manning, V. A., Pandelova, I., Betts, M. F., and Martinez, J. P. 2010. Host-selective toxins, Ptr ToxA and Ptr ToxB, as necrotrophic effectors in the Pyrenophora tritici-repentis-wheat interaction. New Phytol. 187:911-919.

Conesa, A., Götz, S., García-Gómez, J. M., Terol, J., Talón, M., and Robles, M. 2005. Blast2GO: A universal tool for annotation, visualization and analysis in functional genomics research. Bioinformatics 21:3674-3676.

Cook, D. E., Mesarich, C. H., and Thomma, B. P. H. J. 2015. Understanding plant immunity as a surveillance system to detect invasion. Annu. Rev. Phytopathol. 53:541-563.

Cox, M. P., Peterson, D. A., and Biggs, P. J. 2010. SolexaQA: At-a-glance quality assessment of Illumina second-generation sequencing data. BMC Bioinformatics 11:485.

Csárdi, G., and, T. 2006. The igraph software package for complex network research. InterJournal Complex Systems.

De Cremer, K., Mathys, J., Vos, C., Froenicke, L., Michelmore, R. W., Cammue, B. P. A., and De Coninck, B. 2013. RNAseq-based transcriptome analysis of Lactuca sativa infected by the fungal necrotroph Botrytis cinerea. Plant Cell Environ. 36:1992-2007. 
Denoux, C., Galletti, R., Mammarella, N., Gopalan, S., Werck, D., De Lorenzo, G., Ferrari, S., Ausubel, F. M., and Dewdney, J. 2008. Activation of defense response pathways by OGs and Flg22 elicitors in Arabidopsis seedlings. Mol. Plant 1:423-445.

Denton-Giles, M., Bradshaw, R. E., and Dijkwel, P. P. 2013. Ciborinia camelliae (Sclerotiniaceae) induces variable plant resistance responses in selected species of Camellia. Phytopathology 103:725-732.

Fan, Z., Li, J., Li, X., Wu, B., Wang, J., Liu, Z., and Yin, H. 2015. Genomewide transcriptome profiling provides insights into floral bud development of summer-flowering Camellia azalea. Sci. Rep. 5:9729.

Ferrari, S., Plotnikova, J. M., De Lorenzo, G., and Ausubel, F. M. 2003. Arabidopsis local resistance to Botrytis cinerea involves salicylic acid and camalexin and requires EDS4 and PAD2, but not SID2, EDS5 or PAD4. Plant J. 35:193-205.

Gilbert, B. M., and Wolpert, T. J. 2013. Characterization of the LOV1mediated, victorin-induced, cell-death response with virus-induced gene silencing. Mol. Plant-Microbe Interact. 26:903-917.

Glazebrook, J. 2005. Contrasting mechanisms of defense against biotrophic and necrotrophic pathogens. Annu. Rev. Phytopathol. 43:205-227.

Haas, B. J., Papanicolaou, A., Yassour, M., Grabherr, M., Blood, P. D., Bowden, J., Couger, M. B., Eccles, D., Li, B., Lieber, M., MacManes, M. D., Ott, M., Orvis, J., Pochet, N., Strozzi, F., Weeks, N., Westerman, R., William, T., Dewey, C. N., Henschel, R., LeDuc, R. D., Friedman, N., and Regev, A. 2013. De novo transcript sequence reconstruction from RNA-seq using the Trinity platform for reference generation and analysis. Nat. Protoc. 8:1494-1512.

Hahlbrock, K., Bednarek, P., Ciolkowski, I., Hamberger, B., Heise, A., Liedgens, H., Logemann, E., Nürnberger, T., Schmelzer, E., Somssich, I. E., and Tan, J. 2003. Non-self recognition, transcriptional reprogramming, and secondary metabolite accumulation during plant/pathogen interactions. Proc. Natl. Acad. Sci. U.S.A. 100 (Suppl 2):14569-14576.

Han, G. Z. 2017. Evolution of jasmonate biosynthesis and signaling mechanisms. J. Exp. Bot. 68:1323-1331.

Hao, X., Horvath, D. P., Chao, W. S., Yang, Y., Wang, X., and Xiao, B. 2014. Identification and evaluation of reliable reference genes for quantitative real-time PCR analysis in tea plant (Camellia sinensis (L.) O. Kuntze). Int. J. Mol. Sci. 15:22155-22172.

Hara, K. 1919. A sclerotial disease of camellia (Camellia japonica). Dainippon Sanrin Kaiho. 436:29-31.

Ho, C.-L., Wu, Y., Shen, H. B., Provart, N. J., and Geisler, M. 2012. A predicted protein interactome for rice. Rice (N. Y.) 5:15.

Höll, J., Vannozzi, A., Czemmel, S., D’Onofrio, C., Walker, A. R., Rausch, T., Lucchin, M., Boss, P. K., Dry, I. B., and Bogs, J. 2013. The R2R3MYB transcription factors MYB14 and MYB15 regulate stilbene biosynthesis in Vitis vinifera. Plant Cell 25:4135-4149.

Huot, B., Yao, J., Montgomery, B. L., and He, S. Y. 2014. Growth-defense tradeoffs in plants: A balancing act to optimize fitness. Mol. Plant 7: 1267-1287.

Ingle, R. A., and Roden, L. C. 2014. Circadian Regulation of Plant Immunity to Pathogens. Pages 273-283 in: Plant Circadian Networks: Methods and Protocols. D. Staiger, ed. Humana Press, New York.

Jayaswall, K., Mahajan, P., Singh, G., Parmar, R., Seth, R., Raina, A., Swarnkar, M. K., Singh, A. K., Shankar, R., and Sharma, R. K. 2016. Transcriptome analysis reveals candidate genes involved in blister blight defense in tea (Camellia sinensis (L) Kuntze). Sci. Rep. 6:30412.

Kabbage, M., Yarden, O., and Dickman, M. B. 2015. Pathogenic attributes of Sclerotinia sclerotiorum: Switching from a biotrophic to necrotrophic lifestyle. Plant Sci. 233:53-60.

Katagiri, F., and Tsuda, K. 2010. Understanding the plant immune system. Mol. Plant-Microbe Interact. 23:1531-1536.

Kohn, L. M., and Nagasawa, E. 1984. A taxonomic reassessment of Sclerotinia camelliae Hara (=Ciborinia camelliae Kohn), with observations on flower blight in Japan. Trans. Mycol. Soc. Jpn. 25:149-161.

König, S., Feussner, K., Kaever, A., Landesfeind, M., Thurow, C., Karlovsky, P., Gatz, C., Polle, A., and Feussner, I. 2014. Soluble phenylpropanoids are involved in the defense response of Arabidopsis against Verticillium longisporum. New Phytol. 202:823-837.

Langmead, B., and Salzberg, S. L. 2012. Fast gapped-read alignment with Bowtie 2. Nat. Methods 9:357-359.

Li, W., and Godzik, A. 2006. Cd-hit: A fast program for clustering and comparing large sets of protein or nucleotide sequences. Bioinformatics 22:1658-1659.

Li, X., Zhang, Y., Huang, L., Ouyang, Z., Hong, Y., Zhang, H., Li, D., and Song, F. 2014. Tomato SlMKK2 and SlMKK4 contribute to disease resistance against Botrytis cinerea. BMC Plant Biol. 14:166.

Liebrand, T. W. H., van den Burg, H. A., and Joosten, M. H. A. J. 2014. Two for all: Receptor-associated kinases SOBIR1 and BAK1. Trends Plant Sci. 19:123-132.
Love, M. I., Anders, S., and Huber, W. 2014. Differential analysis of count data-The DESeq2 package. Intrexon Bioinformatics Germany $\mathrm{GmbH}$, Munich.

Lu, H., Zhang, J., Yang, Y., Yang, X., Xu, B., Yang, W., Tong, T., Jin, S., Shen, C., Rao, H., Li, X., Lu, H., Fuller, D. Q., Wang, L., Wang, C., Xu, D., and $\mathrm{Wu}$, N. 2016. Earliest tea as evidence for one branch of the Silk Road across the Tibetan Plateau. Sci. Rep. 6:18955.

Maere, S., Heymans, K., and Kuiper, M. 2005. BiNGO: A Cytoscape plugin to assess overrepresentation of gene ontology categories in biological networks. Bioinformatics 21:3448-3449.

Mao, G., Meng, X., Liu, Y., Zheng, Z., Chen, Z., and Zhang, S. 2011. Phosphorylation of a WRKY transcription factor by two pathogenresponsive MAPKs drives phytoalexin biosynthesis in Arabidopsis. Plant Cell 23:1639-1653.

Martin, M. 2011. Cutadapt removes adapter sequences from highthroughput sequencing reads. EMBnet. J. 17:10-12.

Mendiburu, F., and Simon, R. 2015. Agricolae-Ten years of an open source statistical tool for experiments in breeding, agriculture and biology. PeerJ Prepr.

Mondal, T. K. 2011. Camellia. Pages 15-39 in: Wild Crop Relatives: Genomic and Breeding Resources. C. Kole, ed. Springer, Berlin, Germany.

Musungu, B., Bhatnagar, D., Brown, R. L., Fakhoury, A. M., and Geisler, M. 2015. A predicted protein interactome identifies conserved global networks and disease resistance subnetworks in maize. Front. Genet. 6:201.

Pusztahelyi, T., Holb, I. J., and Pócsi, I. 2015. Secondary metabolites in fungus-plant interactions. Front. Plant Sci. 6:573.

R Development Core Team. 2017. R: A language and environment for statistical computing. R Foundation for Statistical Computing, Vienna, Austria.

Ruijter, J. M., Ramakers, C., Hoogaars, W. M. H., Karlen, Y., Bakker, O., van den Hoff, M. J., and Moorman, A. F. 2009. Amplification efficiency: Linking baseline and bias in the analysis of quantitative PCR data. Nucleic Acids Res. 37:e45.

Saracchi, M., Locati, D., Colombo, E. M., and Pasquali, M. 2018. Updates on Ciborinia camelliae, the causal agent of camellia flower blight. J. Plant Pathol. 101:215-223.

Schindelin, J., Arganda-Carreras, I., Frise, E., Kaynig, V., Longair, M., Pietzsch, T., Preibisch, S., Rueden, C., Saalfeld, S., Schmid, B., Tinevez, J. Y., White, D. J., Hartenstein, V., Eliceiri, K., Tomancak, P., and Cardona, A. 2012. Fiji: An open-source platform for biological-image analysis. Nat. Methods 9:676-682.

Shannon, P., Markiel, A., Ozier, O., Baliga, N. S., Wang, J. T., Ramage, D., Amin, N., Schwikowski, B., and Ideker, T. 2003. Cytoscape: A software environment for integrated models of biomolecular interaction networks. Genome Res. 13:2498-2504.

Simão, F. A., Waterhouse, R. M., Ioannidis, P., Kriventseva, E. V., and Zdobnov, E. M. 2015. BUSCO: Assessing genome assembly and annotation completeness with single-copy orthologs. Bioinformatics 31 : 3210-3212.

Szklarczyk, D., Morris, J. H., Cook, H., Kuhn, M., Wyder, S., Simonovic, M., Santos, A., Doncheva, N. T., Roth, A., Bork, P., Jensen, L. J., and von Mering, C. 2017. The STRING database in 2017: Quality-controlled protein-protein association networks, made broadly accessible. Nucleic Acids Res. 45 (D1):D362-D368.

Taylor, C. H., and Long, P. G. 2000. Review of literature on camellia flower blight caused by Ciborinia camelliae. New Zeal. J. Crop Hortic. Sci. 28: 123-138.

Toruño, T. Y., Shen, M., Coaker, G., and Mackey, D. 2019. Regulated disorder: Posttranslational modifications control the RIN4 plant immune signaling hub. Mol. Plant-Microbe Interact. 32:56-64.

Trapnell, C., Roberts, A., Goff, L., Pertea, G., Kim, D., Kelley, D. R., Pimentel, H., Salzberg, S. L., Rinn, J. L., and Pachter, L. 2012. Differential gene and transcript expression analysis of RNA-seq experiments with TopHat and Cufflinks. Nat. Protoc. 7:562-578.

Veloso, J., and van Kan, J. A. L. 2018. Many shades of grey in Botrytis-host plant interactions. Trends Plant Sci. 23:613-622.

Wang, K., Liao, Y., Kan, J., Han, L., and Zheng, Y. 2015. Response of direct or priming defense against Botrytis cinerea to methyl jasmonate treatment at different concentrations in grape berries. Int. J. Food Microbiol. 194:32-39.

Wang, X., Jiang, N., Liu, J., Liu, W., and Wang, G.-L. 2014. The role of effectors and host immunity in plant-necrotrophic fungal interactions. Virulence 5:722-732.

Wang, Y., Chantreau, M., Sibout, R., and Hawkins, S. 2013. Plant cell wall lignification and monolignol metabolism. Front. Plant Sci. 4:220.

Wang, Y., Hao, X., Lu, Q., Wang, L., Qian, W., Li, N., Ding, C., Wang, X., and Yang, Y. 2018a. Transcriptional analysis and histochemistry reveal 
that hypersensitive cell death and $\mathrm{H}_{2} \mathrm{O}_{2}$ have crucial roles in the resistance of tea plant (Camellia sinensis (L.) O. Kuntze) to anthracnose. Hortic. Res. 5:18.

Wang, Y., Schuck, S., Wu, J., Yang, P., Döring, A. C., Zeier, J., and Tsuda, K. 2018b. A MPK3/6-WRKY33-ALD1-pipecolic acid regulatory loop contributes to systemic acquired resistance. Plant Cell 30:2480-2494.

Warnes, G. R., Bolker, B., Bonebakker, L., Gentleman, R., Liaw, W. H. A., Lumley, T., Maechler, M., Magnusson, A., Moeller, S., Schwartz, M., and Venables, B. 2019. gplots: Various R programming tools for plotting data. R Foundation for Statistical Computing, Vienna, Austria.

Williams, B., Kabbage, M., Kim, H. J., Britt, R., and Dickman, M. B. 2011. Tipping the balance: Sclerotinia sclerotiorum secreted oxalic acid suppresses host defenses by manipulating the host redox environment. PLoS Pathog. 7:e1002107.

Wu, J., Zhao, Q., Yang, Q., Liu, H., Li, Q., Yi, X., Cheng, Y., Guo, L., Fan, C., and Zhou, Y. 2016. Comparative transcriptomic analysis uncovers the complex genetic network for resistance to Sclerotinia sclerotiorum in Brassica napus. Sci. Rep. 6:19007.

Xia, E. H., Jiang, J. J., Huang, H., Zhang, L. P., Zhang, H. B., and Gao, L. Z. 2014. Transcriptome analysis of the oil-rich tea plant, Camellia oleifera, reveals candidate genes related to lipid metabolism. PLoS One 9:e104150.
Xu, L., Zhu, L., Tu, L., Liu, L., Yuan, D., Jin, L., Long, L., and Zhang, X. 2011. Lignin metabolism has a central role in the resistance of cotton to the wilt fungus Verticillium dahliae as revealed by RNA-Seq-dependent transcriptional analysis and histochemistry. J. Exp. Bot. 62:5607-5621.

Yao, Q.-Y., Huang, H., Tong, Y., Xia, E.-H., and Gao, L.-Z. 2016. Transcriptome analysis identifies candidate genes related to triacylglycerol and pigment biosynthesis and photoperiodic flowering in the ornamental and oil-producing plant, Camellia reticulata (Theaceae). Front. Plant Sci. 7:163.

Zhu, W., Ronen, M., Gur, Y., Minz-Dub, A., Masrati, G., Ben-Tal, N., Savidor, A., Sharon, I., Eizner, E., Valerius, O., Braus, G. H., Bowler, K., Bar-Peled, M., and Sharon, A. 2017. BcXYG1, a secreted xyloglucanase from Botrytis cinerea, triggers both cell death and plant immune responses. Plant Physiol. 175:438-456.

Zhu, W., Wei, W., Fu, Y., Cheng, J., Xie, J., Li, G., Yi, X., Kang, Z., Dickman, M. B., and Jiang, D. 2013. A secretory protein of necrotrophic fungus Sclerotinia sclerotiorum that suppresses host resistance. PLoS One 8:e53901.

Zhu, Z., and Tian, S. 2012. Resistant responses of tomato fruit treated with exogenous methyl jasmonate to Botrytis cinerea infection. Sci. Hortic. (Amsterdam) 142:38-43. 